\title{
Hartree-Fock Multiplet Strengths for K I, Ca II, and Sc III*
}

\author{
A. W. Weiss \\ Institute for Basic Standards, National Bureau of Standards, Washington, D.C. 20234
}

(December 14, 1966)

\begin{abstract}
Analytical approximations to Hartree-Fock wavefunctions have been computed for the $4 s, 4 p$, and $3 d$ states of $\mathrm{KI}$, Ca II, and Sc III. Multiplet strengths for the $4 s-4 p$ and $4 p-3 d$ transitions in these ions are also computed. Examination of the results indicate that relaxation of the core in the field of the series electron accounts for a large portion of the core polarization effect, at least for the $3 d$ level. Serious cancellation is found to invalidate the dipole velocity results of the $4 p-3 d$ transition in CaII.
\end{abstract}

Key Words: Multiplet strength, self-consistent field, term values, wavefunctions.

\section{Introduction}

In view of the astrophysical importance of singly ionized calcium (Ca II), it is not surprising that a number of calculations should have been made for the most important transition integrals, notably for the $4 s-4 p$ and $4 p-3 d$ transitions. The current status of these calculations is summarized in the paper by Douglas and Garstang [1962]. Attention is drawn there to the still rather large uncertainty in the $4 p-3 d$ transition, as indicated by the scatter in the computed values, as well as the discrepancy between the length and velocity forms, where they have been calculated.

In view of the basic simplicity of the spectrum, it seems reasonable to expect the self-consistent field model to provide a fairly good representation of the ion and, perhaps, shed some light on this problem. This paper, therefore, reports on a study of the HartreeFock approximation as applied to Ca II. Hartree-Fock wavefunctions, and the accompanying transitions, are computed for the $4 s, 4 p$, and $3 d$ levels. To better assess the results, the same calculations were done on the neighboring isoelectronic systems, neutral potassium (K I) and doubly ionized scandium (Sc III).

All of these calculations were done by the expansion method, using the University of Chicago program for the IBM 7094 computer [Roothaan and Bagus, 1963].

\section{Review of Theory}

The configuration for members of the potassium isoelectronic sequence consists of a closed shell core plus a single series electron, i.e.,

$$
(1 s)^{2}(2 s)^{2}(2 p)^{6}(3 s)^{2}(3 p)^{6} n l,
$$

*This research is a part of project DEFENDER, sponsored by the Advanced Research Projects Agency, Department of Defense, through the Office of Naval Research. and, here, $n l=4 s, 4 p$, and $3 d$. In an independent particle model, the total wave function is a single determinant of one-electron functions (orbitals) with each electron assigned to a single function in accordance with the aufbau principle. Application of the variational principle to this type of trial function then leads to the Hartree-Fock self-consistent field (SCF) equations for the orbitals,

$$
F_{i}(\varphi) \varphi_{i}=\epsilon_{i} \varphi_{i}, \varphi_{i}=1 s, 2 s, \ldots, 3 p, n l .
$$

$F_{i}$, the Fock operator, has the form of a one-electron Hamiltonian whose potential represents the average field of all the other electrons of the atom as seen by an electron in the $i$ th orbital. There is a different equation for all the occupied orbitals, and the usual procedure is to solve this set of equations iteratively until the entire set of orbitals is self-consistent.

This brief description of the SCF procedure is not entirely out of place, since this is the point where the present calculations differ from all those previously done on Ca II. In Hartree's original work, both with and without exchange (Hartree and Hartree, 1938), the core functions were carried over directly from the SCF calculations on the core ion, $\mathrm{Ca}^{+2}$, and only the last of the eqs (1) were solved for the series electron. The same is true of the "core polarization" calculations, where a term representing polarization of the core by the outer electron is added onto the potential in the Fock operator. Neglecting the relaxation of the core is usually a reasonably good approximation for alkali type atoms and ions, since the series electron is normally well separated from the core; e.g., the $K$-shell of $\mathrm{Li}$ and $\mathrm{Li}^{+}$are essentially the same. However, as will be seen, this spatial separation is not always achieved for potassium-like systems, with some interesting consequences. 
The multiplet strength for an electric dipole transition $n l \rightarrow n^{\prime} l^{\prime}$ is given by

$$
S=\mathscr{S} \sigma^{2}
$$

where $\mathscr{S}$ is an angular factor readily available in literature tabulations [Allen, 1963], and $\sigma$ is the transition integral. If the core functions don't change significantly for the two states, $\sigma$ is simply given by

$$
\sigma=\left(4 l_{>}^{2}-1\right)^{-1 / 2} \int_{0}^{\infty} d r r^{3} R_{n l} R_{n^{\prime} l^{\prime}},
$$

where $l_{>}$is the greater of the two $l$-values and $R_{n l}$ is the radial function for the $n l$-electron. If the core changes enough to make the nonorthonormality of the orbitals from the two states significant, then $\sigma$ is altered by the inclusion of nonorthogonality and exchange terms. For example, for the $4 p-3 d$ transition $\sigma$ would become

$$
\sigma=\sigma(4 p, 3 d)-\langle 4 p \mid 3 p\rangle \sigma(3 p, 3 d)
$$

where $\langle 4 p \mid 3 p\rangle$ is an overlap integral. While this change of the core orbitals from excited state to excited state is normally small for alkali-like systems, it does make some difference in a few cases, which will be discussed as they arise.

An alternative form of the transition integral (3) is the dipole velocity form [Chandrasekhar, 1945; Bethe and Salpeter, 1957], which is given by

$$
\begin{aligned}
\sigma=\frac{1}{\Delta E}\left(4 l_{>}^{2}-1\right)^{-1 / 2} \int_{0}^{\infty} d r r\left[R_{n l}^{\cdot}\right. & \frac{d}{d r}\left(r R_{n^{\prime} l^{\prime}}\right) \\
& \left. \pm l_{>} R_{n l} R_{n^{\prime} l^{\prime}}\right]
\end{aligned}
$$

where $\Delta E$ is the energy difference of the transition, and the upper sign is taken if $l^{\prime}$ is $l_{>}$and the lower if $l^{\prime}$ is $l_{<}$. While these two forms must give the same value for exact wavefunctions, they need not, and usually do not agree exactly for approximate wavefunctions. Although their agreement is only a necessary and not sufficient condition, the relative disparity does provide another handle on assessing the reliability of a calculation. For alkali-like systems, where an independent particle model should be particularly good, one might attach a little more significance to the length-velocity agreement. In fact, one of the problems here is the fact that a number of independent particle type of calculations give a wide discrepancy between the length and velocity forms for the $4 p-3 d$ transition in Ca II.

\section{Results and Discussion}

Analytical approximations to the Hartree-Fock wavefunctions were computed for the $4 s, 4 p$, and $3 d$ states of KI, Ca II, and Sc III [Roothaan and Bagus, 1963]. Based on comparisons with other calculations, where available [Clementi, 1965; Synek and Roothaan, 1966], and calculations with smaller basis sets, they are estimated to be accurate to about 3 decimal places; i.e., there may be a deviation from the true Hartree-Fock function of about 0.001 . The computed total energies are given, in atomic units, ${ }^{1}$ in table 1 , where the spacing with respect to the ground state is also computed and compared with the spectroscopically observed level

\begin{tabular}{|c|c|c|c|c|}
\hline \multicolumn{2}{|r|}{ Atom or ion } & \multirow{2}{*}{$\begin{array}{l}\text { Total energy } \\
\text { Hartree-Fock }\end{array}$} & \multicolumn{2}{|c|}{$\begin{array}{l}\text { Energy relative to } \\
\text { ground state }\end{array}$} \\
\hline & & & Hartree-Fock & Observed ${ }^{a}$ \\
\hline $\mathrm{K}_{\mathrm{I}}$ & $\begin{array}{r}3 p^{6} 4 s^{2} \mathrm{~S} \ldots \ldots \ldots \ldots \ldots \\
4 p^{2} \mathrm{P} \ldots \ldots \ldots \ldots \ldots \\
3 d^{2} \mathrm{D} \ldots \ldots \ldots \ldots \ldots\end{array}$ & $\begin{array}{l}-599.1646 \\
-599.1130 \\
-599.0755\end{array}$ & $\begin{array}{l}0.0 \\
.0516 \\
.0891\end{array}$ & $\begin{array}{l}0.0 \\
.0593 \\
.0981\end{array}$ \\
\hline Ca II & $\begin{array}{r}3 p^{6} 4 s^{2} \mathrm{~S} \ldots \ldots \ldots \ldots \ldots \\
3 d^{2} \mathrm{D} \ldots \ldots \ldots \ldots \ldots \\
4 p^{2} \mathrm{P} \ldots \ldots \ldots \ldots \ldots\end{array}$ & $\begin{array}{l}-676.5698 \\
-676.4981 \\
-676.4635\end{array}$ & $\begin{array}{l}0.0 \\
.0717 \\
.1063\end{array}$ & $\begin{array}{l}0.0 \\
.0624 \\
.1155\end{array}$ \\
\hline Sc III & $\begin{array}{r}3 p^{6} 3 d^{2} \mathrm{D} \ldots \ldots \ldots \ldots \ldots \\
4 s^{2} \mathrm{~S} \ldots \ldots \ldots \ldots \ldots \\
4 p^{2} \mathrm{P} \ldots \ldots \ldots \ldots \ldots\end{array}$ & $\begin{array}{l}-759.0921 \\
-758.9805 \\
-758.8222\end{array}$ & $\begin{array}{l}0.0 \\
.1116 \\
.2699\end{array}$ & $\begin{array}{l}0.0 \\
.1158 \\
.2839\end{array}$ \\
\hline
\end{tabular}
positions [Moore 1949]. The wavefunctions themselves are given in the appendix.

TABLE 1. Computed and observed energies (in a.u.) for the potassium isoelectronic sequence

a Moore, 1949.

The positions of the energy levels are computed with fair accuracy - the errors range from 0.1 to $0.3 \mathrm{eV}$. This is about the level of accuracy one can reasonably expect from the Hartree-Fock approximation for such systems. Differences in the correlation energy between the outer electron and the core can easily amount to the order of $0.1 \mathrm{eV}$. This correlation error can be taken into account by configuration mixing, of course. Alternatively, since it is due to the deviations from the Hartree-Fock averaging over electronic interactions, one can also include the effect by introducing a polarization potential into the series electron equation, which represents the "instantaneous distortion" of the core from spherical symmetry due to the outer electron. As expected, polarization potential calculations do indeed give an improved term scheme [Douglas, 1956, Trefftz and Biermann, 1952]. As mentioned earlier, however, all the calculations done on Ca II used the frozen core approximation, with orbitals taken from Ca III, and it should be somewhat instructive to look more closely at the effects of relaxation.

Table 2 shows the location of the principal maxima of the series electron orbitals compared with that of the $3 p$ core function. The $3 p$ value given here is that of the argon-like ion, the change in the principal maximum for potassium-like systems being small. What one sees, of course, is the well-known phenomenon of the $3 d$ electron being buried in the body of the

\footnotetext{
${ }^{1}$ Atomic units are used throughout this paper. Energy (in a.u.) $=2 R_{M}$, where $R_{M}$ is the appropriate reduced mass Rydberg, and the length unit $=0.52915 \AA$.
} 
atom as you move along the isoelectronic sequence in the direction of increasing positive ionicity. Only for neutral potassium can the $3 d$ electron be thought of as being well separated from the core, and for doubly ionized scandium, it occupies essentially the same region of space as the $3 p$ core electrons.

TABLE 2. Location of principle maxima (in a.u.) for potassium-like wavefunctions

\begin{tabular}{l|c|c|c|c}
\hline \hline & $3 p$ & $4 s$ & $4 p$ & $3 d$ \\
\hline & & & & \\
K I I............. & 1.16 & 4.34 & 5.92 & 8.30 \\
Ca II ........... & 1.05 & 3.25 & 3.96 & 1.46 \\
Sc III......... & 0.97 & 2.72 & 3.15 & 1.10 \\
\hline
\end{tabular}

While the relaxation of the $3 p$ function is small, it introduces enough of a change in the field seen by the series electron to make a significant change over the frozen core approximation. This is shown in figure 1 , where the Hartree-Fock $3 d$ for Ca II is compared with the frozen core SCF with exchange. Also shown is the $3 d$ function of Douglas and Garstang [1962] computed with a polarization term in the potential, which agrees remarkably well with the Hartree-Fock function, suggesting that, to a large extent, the polarization is serving to mock up the core relaxation effect. The difference between the Hartree-Fock and polarization $3 d$ is now comparable to the changes found by Douglas for the $4 s$ and $4 p$ orbitals.

The Hartree-Fock multiplet strength data for the $4 s-4 p$ and $4 p-3 d$ transitions in these ions are given in table 3 . The area ratio column refers to the ratio of the positive and negative parts of the principal transition integral for either eq (3) or (5), which, of course, is a measure of the degree to which cancellation is important. As can be seen, the agreement of the length and velocity values ranges from fairly good to excellent the mean of the two \pm 20 percent easily encompasses both values. The sole exception to this observation is the $4 p-3 d$ transition in Ca II, which stands out like the proverbial sore thumb. It seems quite clear that cancellation in the velocity integral is giving a wrong

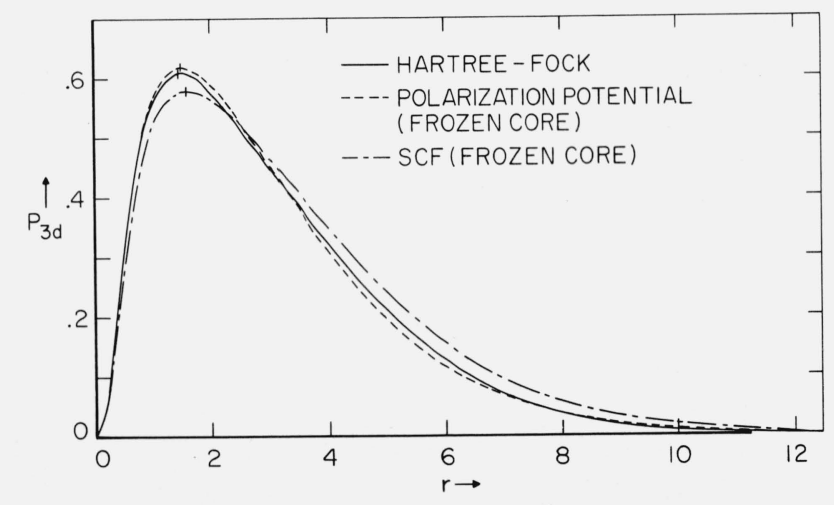

Figure 1. Radial 3d-functions for Ca II.

$$
\mathrm{P}_{3 d}=r \mathrm{R}_{3 d} \text {. }
$$

result, and, since this makes the principal transition integral small, the core nonorthogonality effects are accentuated in this case. The inclusion in $\sigma$ of an exchange term as shown in (4) changes $\mathrm{S}$ from 5.0 to 9.7. In the case of Sc III the velocity form of $\mathrm{S}$ is changed from 3.89 to 4.86 . This cancellation has also been found in a recent calculation by Garstang and Hill [Garstang and Hill, 1966] of the velocity integral for Hartree's SCF functions with frozen core.

TABLE 3. Hartree-Fock results for the potassium isoelectronic sequence transitions

\begin{tabular}{|c|c|c|c|c|}
\hline Transition & $\begin{array}{l}\text { Wave- } \\
\text { length }\end{array}$ & Type & $\begin{array}{l}\text { Multiplet } \\
\text { strength }\end{array}$ & Area ratio \\
\hline \multirow{3}{*}{$\begin{array}{c}4 s-4 p \\
\mathrm{~K}_{\mathrm{I}} \ldots \ldots \ldots \ldots \ldots\end{array}$} & \multirow{3}{*}{$\stackrel{\AA}{\AA}$} & & & \\
\hline & & Length..... & 62.1 & $2.8 \times 10^{4}$ \\
\hline & & Velocity.. & 44.9 & 17.3 \\
\hline \multirow[t]{2}{*}{ Ca II ............ } & \multirow[t]{2}{*}{3945} & Length..... & 30.7 & $1.9 \times 10^{4}$ \\
\hline & & Velocity.... & 24.6 & 9.16 \\
\hline \multirow[t]{2}{*}{ Sc III............ } & \multirow[t]{2}{*}{2711} & Length..... & 20.0 & $2.7 \times 10^{4}$ \\
\hline & & Velocity.. & 16.8 & 6.72 \\
\hline \multirow{3}{*}{$\begin{array}{c}4 p-3 d \\
\mathrm{~K} \mathrm{I} \ldots \ldots \ldots \cdots \cdots\end{array}$} & \multirow{3}{*}{11743} & & & \\
\hline & & Length..... & 224 & 933 \\
\hline & & Velocity ... & 218 & 9.86 \\
\hline \multirow[t]{2}{*}{ Ca II ............ } & \multirow[t]{2}{*}{8581} & Length..... & 21.6 & 29.2 \\
\hline & & Velocity ... & 5.0 & 1.2 \\
\hline \multirow[t]{2}{*}{ Sc III............ } & \multirow[t]{2}{*}{1605} & Length..... & 5.51 & 11.4 \\
\hline & & Velocity... & 3.89 & 1.94 \\
\hline
\end{tabular}

Finally, table 4 collects together for comparison purposes the transition integral calculations on Ca II by a number of other authors. It can be seen that there is substantial agreement among all these calculations, including the present Hartree-Fock values. Furthermore, it appears that, for the $4 p-3 d$ transition, the effect of relaxing the core in the field of the series electron (Hartree-Fock) is essentially the same as including a polarization potential with a frozen core (as illustrated by both the Douglas and Garstang and Trefftz and Biermann results). The Hartree-Hartree value is for a frozen core without polarization.

\section{Conclusions}

The first conclusions to be drawn concern the wavefunctions. It seems clear, a priori, that neglecting

TABLE 4. Comparison of calculations of the transition integral $\left(\sigma^{2}\right)$ for calcium II

\begin{tabular}{l|r|r|r|c}
\hline \hline & \multicolumn{2}{|c|}{$4 s-4 p$} & \multicolumn{2}{c}{$4 p-3 d$} \\
\cline { 2 - 5 } & Length & Velocity & Length & Velocity \\
\cline { 2 - 5 } & & & & \\
Hartree-Fock (this paper)........ & 5.13 & 4.09 & 0.36 & 0.084 \\
Douglas \& Garstang 1962........ & 4.74 & 4.45 & .35 & .095 \\
Trefftz \& Biermann 1952 ........ & 4.76 & 4.47 & .31 & .11 \\
Hartree \& Hartree 1938........ & 5.15 & 4.1 & .46 & .005 \\
Bates \& Damgaard 1949........ & 4.38 & & .25 & \\
\hline
\end{tabular}


core relaxation when there is a significant amount of penetration should have a marked effect on the wavefunctions, and the present calculations bear this out for the rather simple case of an alkali-like spectrum. It is interesting to note that the ad hoc introduction of a polarization potential in such a case serves to reproduce the bulk of the relaxation effect.

As for the multiplet strengths themselves, the values computed here are probably fairly reliable. There are three considerations on which this is based: (1) the overall agreement with a number of other authors on Ca II, (2) the generally good agreement of the length and velocity forms of the multiplet strengths, and (3) the fundamental soundness of an independent particle model for such a system. It is suggested, therefore, that one should take the mean of the length and velocity results of table 3 , with an uncertainty of about 25 percent, which encompasses all the directly computed numbers. The sole exception to this, of course, is the $4 p-3 d$ transition of Ca II, where the large cancellations occur for the velocity form. In this case, the length value is probably good to about 25 percent.

\section{Appendix}

The Hartree-Fock orbitals are represented, in the expansion method, as a linear combination of analytical basis functions,

$$
\varphi_{p}=\sum_{i} C_{i}^{p} \chi_{i}
$$

where the $C_{i}^{p}$ are the expansion coefficients and $\chi_{i}$ the basis functions. The basis functions used here are the normalized Slater type orbitals, whose radial parts are of the form,

$$
\chi_{n}=(2 \zeta)^{n+1 / 2}[(2 n) !]^{-1 / 2} r^{n-1} e^{-\zeta r}
$$

when $n$ is an integer, and $\zeta$ is simply a variational parameter.

The detailed results of the present calculations are given in the accompanying tables. Table Al gives the orbital energies, and tables A2 to A4 give the basis function parameters and expansion coefficients.

TABLE Al. Orbital energies

\begin{tabular}{c|c|r|r|r}
\hline \hline & & & & \\
State & Orbital & \multicolumn{1}{c|}{ K I } & \multicolumn{1}{c}{ Ca II } & \multicolumn{1}{c}{ Sc III } \\
\hline & & & & \\
$3 p^{6} 4 s^{2} S$ & $1 s$ & 133.5332 & 149.6095 & 166.7134 \\
& $2 s$ & 14.4901 & 17.0668 & 19.9253 \\
& $3 s$ & 1.7488 & 2.4852 & 3.3591 \\
& $4 s$ & 0.1475 & 0.4163 & 0.7670 \\
& $2 p$ & 11.5194 & 13.8742 & 16.5093 \\
& $3 p$ & 0.9544 & 1.5817 & 2.3462 \\
$3 p^{6} 4 p^{2} P$ & $1 s$ & 133.5873 & 149.6572 & 166.7556 \\
& $2 s$ & 14.5435 & 17.1134 & 19.9658 \\
& $3 s$ & 1.8010 & 2.5290 & 3.3959 \\
& $2 p$ & 11.5736 & 13.9226 & 16.5527 \\
& $3 p$ & 1.0081 & 1.6291 & 2.3885 \\
& $4 p$ & 0.0956 & 0.3095 & 0.6078 \\
$3 p^{63} d^{2} D$ & $1 s$ & 133.6251 & 149.4658 & 166.4673 \\
& $2 s$ & 14.5803 & 16.8979 & 19.6392 \\
& $3 s$ & 1.8357 & 2.3264 & 3.1168 \\
& $2 p$ & 11.6104 & 13.7095 & 16.2301 \\
& $3 p$ & 1.0428 & 1.4403 & 2.1294 \\
& $3 d$ & 0.0581 & 0.3570 & 0.9045 \\
& & & & \\
& & & &
\end{tabular}


TABLE A2. Basis function parameters and expansion coefficients for the $3 \mathrm{p}^{6} 4 \mathrm{~s}^{2} \mathrm{~S}$ state

\begin{tabular}{|c|c|c|c|c|c|c|c|c|c|c|c|c|c|c|c|}
\hline \multicolumn{6}{|c|}{ K I } & \multicolumn{5}{|c|}{ Ca II } & \multicolumn{5}{|c|}{ Sc III } \\
\hline$n$ & $\zeta$ & $1 s$ & $2 s$ & $3 s$ & $4 s$ & $\zeta$ & $1 s$ & $2 s$ & $3 s$ & $4 s$ & $\zeta$ & $1 s$ & $2 s$ & $3 s$ & $4 s$ \\
\hline $\begin{array}{l}1 \\
1 \\
2 \\
2 \\
3 \\
3 \\
3 \\
4 \\
4\end{array}$ & $\begin{array}{c}16.51 \\
28.3 \\
7.85 \\
6.6 \\
20.54 \\
3.55 \\
2.35 \\
1.16 \\
.71\end{array}$ & $\begin{array}{r}0.94593 \\
.11551 \\
-.00193 \\
.00204 \\
-.07064 \\
-.00024 \\
.00008 \\
-.00002 \\
.00001\end{array}$ & $\begin{array}{r}-0.37901 \\
.01461 \\
.39322 \\
.71064 \\
-.02936 \\
.01267 \\
-.00263 \\
.00058 \\
-.00027\end{array}$ & $\begin{array}{r}0.12755 \\
-.00603 \\
.04237 \\
-.52685 \\
.00979 \\
.62787 \\
.52163 \\
.00163 \\
.00016\end{array}$ & $\begin{array}{r}-0.02456 \\
.00115 \\
-.02316 \\
.12159 \\
-.00128 \\
-.15762 \\
-.07810 \\
.46183 \\
.61784\end{array}$ & $\begin{array}{l}17.5 \\
30.31 \\
8.413 \\
7.26 \\
21.85 \\
3.87 \\
2.69 \\
1.59 \\
1.1\end{array}$ & $\begin{array}{r}0.95615 \\
.10429 \\
-.00302 \\
.00290 \\
-.06935 \\
-.00030 \\
.00012 \\
-.00003 \\
.00001\end{array}$ & $\begin{array}{r}-0.38207 \\
.01556 \\
.22229 \\
.87831 \\
-.03115 \\
.01908 \\
-.00615 \\
.00160 \\
-.00072\end{array}$ & $\begin{array}{r}0.13404 \\
-.00644 \\
.16638 \\
-.67436 \\
.01020 \\
.60049 \\
.54961 \\
.00236 \\
.00062\end{array}$ & $\begin{array}{r}-0.03780 \\
.00183 \\
-.05761 \\
.20656 \\
-.00300 \\
-.20050 \\
-.17587 \\
.37627 \\
.70947\end{array}$ & $\begin{array}{c}18.27 \\
32.33 \\
8.6 \\
7.85 \\
22.93 \\
4.3 \\
3.08 \\
1.84 \\
1.414\end{array}$ & $\begin{array}{r}0.96562 \\
.10188 \\
-.00644 \\
.00567 \\
-.07782 \\
-.00020 \\
.00001 \\
.00003 \\
-.00002\end{array}$ & $\begin{array}{r}-0.38648 \\
.01414 \\
.14781 \\
.95158 \\
-.03014 \\
.02492 \\
-.00883 \\
.00254 \\
-.00134\end{array}$ & $\begin{array}{r}0.14080 \\
-.00614 \\
.43430 \\
-.97252 \\
.01013 \\
.51871 \\
.64088 \\
.00429 \\
.00041\end{array}$ & $\begin{array}{r}-0.04766 \\
.00210 \\
-.17477 \\
.36531 \\
-.00359 \\
-.20523 \\
-.26931 \\
.37737 \\
.72176\end{array}$ \\
\hline \multicolumn{6}{|c|}{$2 p$} & \multicolumn{5}{|c|}{$2 p \quad 3 p$} & \multicolumn{5}{|c|}{$2 p \quad 3 p$} \\
\hline $\begin{array}{l}2 \\
2 \\
2 \\
3 \\
3 \\
4\end{array}$ & $\begin{array}{c}6.286 \\
9.92 \\
17.2 \\
3.28 \\
1.565 \\
2.486\end{array}$ & $\begin{array}{r}0.71952 \\
.28940 \\
.01941 \\
.00964 \\
.00094 \\
-.00275\end{array}$ & $\begin{array}{r}-.25478 \\
-.07577 \\
-.00647 \\
.64835 \\
.14319 \\
.34640\end{array}$ & & & $\begin{array}{c}6.784 \\
10.66 \\
18.45 \\
3.59 \\
1.8 \\
2.78\end{array}$ & $\begin{array}{r}.73446 \\
.27464 \\
.01684 \\
.01012 \\
.00093 \\
-.00291\end{array}$ & $\begin{array}{r}-.28229 \\
-.07414 \\
-.00626 \\
.68092 \\
.10055 \\
.35341\end{array}$ & & & $\begin{array}{c}7.342 \\
11.575 \\
19.2 \\
3.95 \\
2.61 \\
3.02\end{array}$ & $\begin{array}{r}.76631 \\
.24083 \\
.01547 \\
-.00034 \\
.04655 \\
-.03876\end{array}$ & $\begin{array}{r}-.31149 \\
-.06541 \\
-.00645 \\
.51232 \\
.69577 \\
-.10589\end{array}$ & & \\
\hline
\end{tabular}

TABLE A3. Basis function parameters and expansion coefficients for the $3 \mathrm{p}^{6} 4 \mathrm{p}{ }^{2} \mathrm{P}$ state

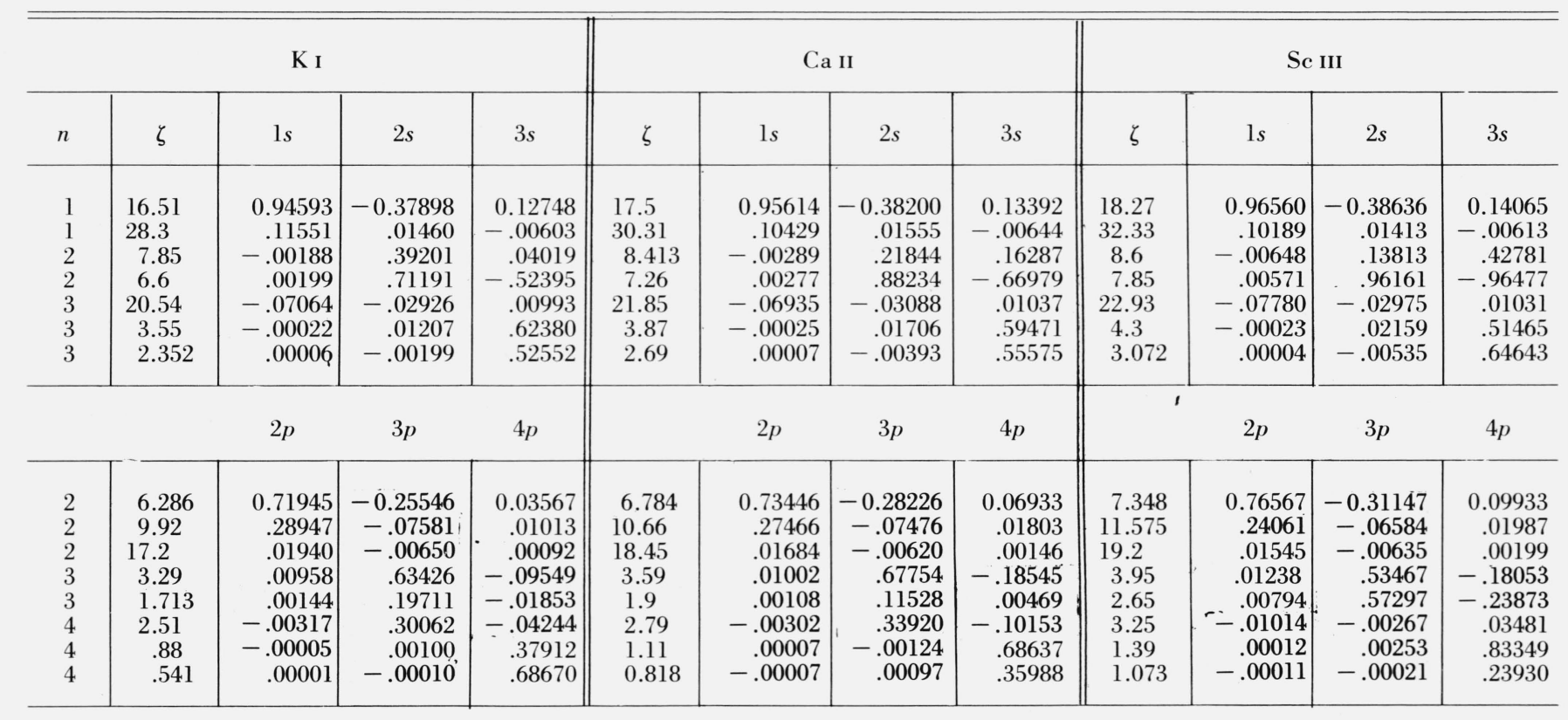


TABLE A4. Basis function parameters and expansion coefficients for the $3 \mathbf{p}^{6} 3 \mathrm{~d}^{2} \mathrm{D}$ state

\begin{tabular}{|c|c|c|c|c|c|c|c|c|c|c|c|c|}
\hline \multicolumn{5}{|c|}{$\mathrm{K}_{\mathrm{I}}$} & \multicolumn{4}{|c|}{ Ca II } & \multicolumn{4}{|c|}{ Sc III } \\
\hline$n$ & $\zeta$ & $1 s$ & $2 s$ & $3 s$ & $\zeta$ & $1 s$ & $2 s$ & $3 s$ & $\zeta$ & $1 s$ & $2 s$ & $3 s$ \\
\hline $\begin{array}{l}1 \\
1 \\
2 \\
2 \\
3 \\
3 \\
3\end{array}$ & $\begin{array}{c}16.51 \\
28.3 \\
7.85 \\
6.6 \\
20.54 \\
3.55 \\
2.347\end{array}$ & $\begin{array}{r}0.94593 \\
.11551 \\
-.00188 \\
.00199 \\
-.07064 \\
-.00022 \\
.00005\end{array}$ & $\begin{array}{r}-0.37899 \\
.01461 \\
.39207 \\
.71187 \\
-.02926 \\
.01201 \\
-.00196\end{array}$ & $\begin{array}{r}0.12748 \\
-.00603 \\
.04193 \\
-.52605 \\
.00981 \\
.62716 \\
.52287\end{array}$ & $\begin{array}{c}17.5 \\
30.31 \\
8.4 \\
7.3 \\
21.85 \\
3.87 \\
2.596\end{array}$ & $\begin{array}{r}0.95618 \\
.10429 \\
-.00315 \\
.00300 \\
-.06938 \\
-.00024 \\
.00006\end{array}$ & $\begin{array}{r}-0.38196 \\
.01553 \\
.18815 \\
.91131 \\
-.03027 \\
.01783 \\
-.00393\end{array}$ & $\begin{array}{r}0.13225 \\
-.00631 \\
.19668 \\
-.69812 \\
.00940 \\
.61535 \\
.53941\end{array}$ & $\begin{array}{c}18.27 \\
32.33 \\
8.6 \\
7.85 \\
22.93 \\
4.28 \\
2.94\end{array}$ & $\begin{array}{r}0.96566 \\
.10188 \\
-.00682 \\
.00602 \\
-.07784 \\
-.00023 \\
.00004\end{array}$ & $\begin{array}{r}-0.38647 \\
.01417 \\
.13839 \\
.96141 \\
-.02962 \\
.02090 \\
-.00460\end{array}$ & $\begin{array}{r}0.13823 \\
-.00603 \\
.42668 \\
-.95457 \\
.00980 \\
.54995 \\
.61398\end{array}$ \\
\hline \multicolumn{5}{|c|}{$2 p$} & \multicolumn{4}{|c|}{$2 p \quad 3 p$} & \multicolumn{4}{|c|}{$2 p \quad 3 p$} \\
\hline $\begin{array}{l}2 \\
2 \\
2 \\
3 \\
3 \\
4\end{array}$ & $\begin{array}{c}6.286 \\
9.92 \\
17.2 \\
3.29 \\
1.67 \\
2.49\end{array}$ & $\begin{array}{r}0.71947 \\
.28947 \\
.01941 \\
.00952 \\
.00120 \\
-.00292\end{array}$ & $\begin{array}{r}-0.25626 \\
-.07533 \\
-.00659 \\
.64124 \\
.16721 \\
.32621\end{array}$ & & $\begin{array}{c}6.784 \\
10.66 \\
18.45 \\
3.58 \\
1.62 \\
2.7\end{array}$ & $\begin{array}{r}0.73405 \\
.27507 \\
.01681 \\
.01013 \\
.00088 \\
-.00264\end{array}$ & $\begin{array}{r}-0.27727 \\
-.07295 \\
-.00615 \\
.67860 \\
.07911 \\
.38395\end{array}$ & & $\begin{array}{c}7.338 \\
11.575 \\
19.2 \\
3.93 \\
2.26 \\
3.12\end{array}$ & $\begin{array}{r}0.76397 \\
.24297 \\
.01516 \\
.01324 \\
.00289 \\
-.00565\end{array}$ & $\begin{array}{r}-0.30422 \\
-.06405 \\
-.00627 \\
.64050 \\
.22938 \\
.25999\end{array}$ & \\
\hline \multicolumn{5}{|c|}{$3 d$} & \multicolumn{4}{|c|}{$3 d$} & \multicolumn{4}{|c|}{$3 d$} \\
\hline $\begin{array}{l}3 \\
3 \\
3 \\
3\end{array}$ & $\begin{array}{l}0.359 \\
1.4 \\
7.88 \\
3.4\end{array}$ & $\begin{array}{r}0.97724 \\
.07898 \\
.00162 \\
.02887\end{array}$ & & & $\begin{array}{l}2.37 \\
1.06 \\
8.72 \\
4.53\end{array}$ & $\begin{array}{r}0.41387 \\
.63671 \\
.01173 \\
.12310\end{array}$ & & & $\begin{array}{l}2.85 \\
1.59 \\
9.26 \\
4.85\end{array}$ & $\begin{array}{r}0.42944 \\
.52111 \\
.01865 \\
.16484\end{array}$ & & 6 \\
\hline
\end{tabular}

\section{References}

Allen, C. W. (1963), Astrophysical Quantities (London: Univ. of London Press).

Bates, D. R., and Damgaard, A. (1949), Phil. Trans. Roy. Soc. A242, 101.

Bethe, H. A., and Salpeter, E. E. (1957), Quantum Mechanics of One-Two-Electron Atoms (New York: Academic Press), pp. 251253.

Chandrasekhar, S. (1945), Astrophys. J. 102, 223.

Clementi, E. (1965), IBM J. Res. Develop. 9, 2.

Douglas, A. S. (1956), Proc. Camb. Phil. Soc. 52, 687.

Douglas, A. S., and Garstang, R. H. (1962), Proc. Camb. Phil. Soc. $\mathbf{5 8}, 377$.
Garstang, R. H., and Hill, S. J. (1966), Publ. Astron. Soc. Pacific, 78,70 .

Hartree, D. R., and Hartree, W. (1938), Proc. Roy. Soc. A164, 167.

Moore, C. E. (1949), Atomic Energy Levels, National Bureau of Standards Circ. 467 (Washington, D.C., Government Printing Office), Vol. 1.

Roothaan, C. C. J., and Bagus, P..S. (1963), Methods in Computational Physics (New York, Academic Press), Vol. II, p. 47.

Synek, M., and Roothaan, C. C. J. (1966), Phys. Rev. 147, 730.

Trefftz, E., and Biermann, L. (1952), Z. Astrophys. 30, 275.

(Paper 71A2-447) 